\title{
A Crise na Biodiversidade e suas Reverberações na Saúde Humana: um Panorama Teórico
}

\author{
The Crisis in Biodiversity and its Reverberations in Human Health: a Theoretical Overview
}

\author{
Lázaro Araújo Santos
}

Universidade Estadual do Sudoeste da Bahia. BA, Brasil.

E-mail: lazaro15_@hotmail.com

\begin{abstract}
Resumo
O declínio da biodiversidade devido as ações humanas têm sido documentadas há muito tempo. Contudo, nas últimas décadas esse declínio tem atingidos valores alarmantes. Como resultado da perda da diversidade biológica observa-se inúmeras consequências, o presente trabalho, enfocara nas consequências sanitárias, e, dessa forma discutirá quais são as principais reverberações que a atual crise na biodiversidade acarreta a saúde humana. Para tanto, foram realizadas buscas bibliográficas em diferentes plataformas acadêmicas. Após análise dos artigos selecionados, inferiu-se que as principais consequências da perda da biodiversidade para saúde humana foram: Aumento no número de doenças zoonóticas; crescimento no número de pessoas que adoeceram direta, ou indiretamente - devido a mudanças climáticas e a emergência e/ou re-emergência de enfermidades provenientes da poluição. Assim, sugere-se que estudos que incorporem as duas dimensões, saúde e biodiversidade, possam ocorrer com maior ênfase, tendo em vista que compreender essa correlação não só nos auxiliará na construção de mecanismo de proteção como, também, poderá ajudar na elaboração de medidas para evitar doenças com potencial pandêmico. Além disso, faz-se necessário a inserção de ações conservacionistas dentro das políticas de saúde global, uma vez que diferentes quadros patológicos são oriundos das modificações que a humanidade vem realizando à biodiversidade.
\end{abstract}

Palavras-chave: Conservação. Diversidade Biológica. Políticas Sanitárias.

\begin{abstract}
Biodiversity decline due to human actions has been documented for a long time. However, in recent decades this decline has reached alarming levels. As a result of the loss of biological diversity, there are numerous consequences, this work will focus on the health consequences, and thus will discuss what are the main reverberations that the current crisis in biodiversity causes to human health. Therefore, bibliographic searches were carried out in different academic platforms. After analyzing the selected articles, it was inferred that the main consequences of the loss of biodiversity for human health were: Increase in the number of zoonotic diseases; growth in the number of people who became ill directly or indirectly due to climate change and the emergence and/or re-emergence of illnesses arising from the emergency. Thus, it is essential that incorporating as two dimensions, health and biodiversity, occur with greater emphasis, considering that understanding this comparison of studies not only in aids in the construction of a protection mechanism, but also can help in the definition of measures to prevent diseases with pandemic potential. In addition, it is necessary to insert conservation actions within global health policies, since different pathological conditions arise from the modification that humanity has been making to biodiversity.
\end{abstract}

Keywords: Conservation. Biological Diversity. Health Policies.

\section{Introdução}

O termo biodiversidade ou diversidade biológica pode ser definido como sendo toda a variedade de organismo, genes e ecossistemas, que compõe a biosfera, englobando, ainda, as paisagens e todos os processos desempenhados nos diferentes níveis - organísmico, genético e ecossistêmico (METZGER; CASATTI, 2006).

Dentre as mais diversas funções e serviços que são realizadas pela diversidade biológica, destacam-se: a produtividade primária; fornecimento de água e ar puros; provisão de diferentes recursos naturais; realização dos ciclos biogeoquímicos; imobilização de materiais tóxicos, tal como o chumbo; regulação climática e proteção contra o surgimento e/ou reaparecimento de inúmeras patologias (GOMES;
NETO; SILVA, 2018; SINISGALLI; SOUZA JUNIOR, 2020)

Vale salientar que serviços e funções ecossistêmicas não são a mesma coisa. Por função entende-se todos os processos realizados pela biodiversidade, enquanto no que concerne os serviços ecossistêmicos, refere-se somente aquelas funções que apresentam, direta ou indiretamente, benefícios aos seres humanos (SIMÕES et al., 2015).

Entretanto, seja no desempenhar das funções, ou na prestação de serviços, faz-se necessário que a biodiversidade esteja em seu estado íntegro, uma vez que todas as ações protagonizada pela diversidade biológica resulta das interações que ocorre no ecossistema (ALHO, 2012). Dessa forma, é de fundamental importância que a estrutura biótica e abiótica se encontre em seu estado de plenitude (ALHO, 2012). 
Contudo, ao ser observados os dados alarmantes de desmatamento e ao aumento vertiginoso da poluição nos diferentes sistemas ecológicos, bem como a extinção em massa causada pelas ações humanas, poderá ser inferido que é justamente na contramão da conservação da biodiversidade que a humanidade caminha, e caminha a passos largos (SANTOS et al., 2019, SOARES et al., 2019).

Como consequência da perda da diversidade, nota-se, mais do que apenas a interrupção dos serviços ecossistêmicos, como também, importantes reverberações em diferentes esferas que compõe a sociedade humana (TRIVELLATO; SANTOS, 2019). Entre as esferas afetadas, destaca-se nesse trabalho a saúde humana.

A Organização Mundial da Saúde (OMS), define saúde como sendo o completo estado de bem-estar físico, mental e social (OMS, 1946). Havendo, portanto, a necessidade de que condições sanitárias, sociais, psicológicas e ambientais sejam supridas a fim de que a população de fato atinja o estado saudável.

Uma das maneiras mais utilizadas para investigar e categorizar como essas condições estão estruturadas dentro da sociedade, é através dos determinantes sociais de saúde. De acordo a Comissão Nacional sobre Determinantes Sociais de Saúde (2006), os determinantes são os fatores sociais, econômicos, culturais, étnicos/raciais, ambientais, psicológicos e comportamentais que influenciam a ocorrência de problemas de saúde e seus fatores de risco na população.

Para além disso, de acordo Camponogara (2012) e Monteiro (2017), é necessário pensar-se em saúde a partir de uma perspectiva global, envolvendo os fatores técnicos e sociais, e os aspectos ambientais. Dado que alterações no meio ambiente acarretam perturbações na dinâmica ecológica, que podem contribuir de maneira decisiva para a manutenção do estado saudável ou para o adoecimento da população (CAMPONOGARA, 2012).

Partindo do princípio de que a saúde, como supracitado, é muito mais que a ausência de doença, necessitando ser levado em consideração aspectos sociais e ambientais, o presente trabalho possui como objetivo apresentar, com base nas publicações acadêmicas recentes, como a destruição da biodiversidade reverbera na condição sanitária da humanidade.

\section{Desenvolvimento}

\subsection{Metodologia}

O presente estudo se caracteriza como uma pesquisa qualitativa, de cunho descritivo e exploratório. Sendo englobado também como uma investigação bibliográfica, haja vista basear-se fundamentalmente nos estudos já publicados (OLLAIK; ZILLER, 2012). Essa investigação se deu entre março de 2021 até julho do mesmo ano.

Dessa forma, a fim de atingir o objetivo apresentado, realizou-se buscas bibliográficas nas seguintes plataformas acadêmicas: Google acadêmico; PubMed; SCIELO.
Utilizando como descritores os termos biodiversidade, destruição, humana, impactos, perda, saúde.

Após a realização das buscas, foram selecionados apenas os trabalhos que estivessem na modalidade de artigo, estando, ainda, disponíveis integralmente de forma gratuita, possuíssem vínculo com alguma instituição de pesquisa brasileira, apresentassem nas palavras chaves e/ou título ao menos três dos descritores utilizados nas buscas e, que houvera sido publicado entre os anos de 2010 e 2020, tal delimitação temporal se deu a fim de nos ater, primordialmente, as discussões mais atualizadas.

Tendo sido efetuadas as buscas e a seleção dos trabalhos, analisou-se as produções seguindo três etapas: i) leitura analítica dos textos, buscando principais impactos da perda da diversidade biológica na saúde, ii) construção de um texto preliminar, concatenando as principais ideias e temáticas apresentadas nos artigos, iii) elaboração final do texto associado a discussões sobre a temática aqui proposta. Vale destacar que a análise seguiu pressuposto de indicados por Patias e Hohendorff (2019).

\subsection{Resultados e discussão}

Realizado o percurso metodológico, foram obtidos 97 trabalhos, dos quais 67 atenderam aos critérios de seleção aqui dispostos. A partir da análise, averiguou-se que os principais impactos ocasionados pelo declínio da diversidade biológica â saúde foram: Aumento no número de doenças zoonóticas; crescimento no número de pessoas que adoeceram direta, ou indiretamente, devido a mudanças climáticas e a emergência e/ou re-emergência de enfermidades provenientes da poluição.

\subsubsection{Aumento no número de zoonoses}

Ao ser analisada as últimas décadas, de todas as doenças que emergiram ou reemergiram, 60 a 70\% foram de origem zoonóticas. Doenças classificadas como zoonose, são aquelas cujo agente patológico consegue trafegar entre o ser humano e outro animal vertebrado, causando, na maioria das vezes, importantes danos a ambos.

Autores como Zanella (2016), Rabelo e Oliveira (2020), Silva (2021) demonstram que muito dessa ascendência no número de zoonose se deve, dentre outros fatores, ao declínio da biodiversidade, pois como consequência desse declínio há, também, a diminuição da heterogeneidade genética que compunha aquele ecossistema. É essa heterogeneidade que impede a ocorrência de um evento realizado pelos patógenos, evento esse denominado de transbordamento.

Transbordamento é um evento, fundamentalmente molecular, no qual um determinado agente patológico, próprios de uma dada espécie, desenvolve, por meio de processos evolutivos, características moleculares que o permite colonizar hospedeiros de outras espécies (AMARAL, 2020).

Em um ambiente que vêm sendo degradado 
constantemente, por exemplo, clareiras abertas por empresas madeireiras, ou, áreas que são usadas para agropecuária intensiva, o declínio da biodiversidade é eminente (FILHO, 2020). Consequentemente, quando o número de animais, ou, de outros organismos que funcionam como hospedeiros para diferentes tipos de patógenos diminui, esses patógenos passam por um processo de seleção, no qual aqueles que conseguirem expandir seus nichos - tipos de hospedeiros -, se sairá bemsucedido (ZANELLA, 2016). Infelizmente, essa nova espécie a ser colonizada, eventualmente, pode ser a espécie humana.

Vale salientar que importantes agentes patogênicos realizaram esse processo, como os causadores do Ebola, da febre de Lassa, da síndrome respiratória do oriente médio, síndrome respiratória aguda grave, síndrome da imunodeficiência adquirida e os causadores da gripe aviária e suína (RIBEIRO et al., 2020, PEREIRA; NARCISO; MANSANO, 2021, SOUZA, 2021).

Além do mais, a diminuição dos habitats e ecossistemas, contribui para que diversas espécies, e, portanto, seus patógenos, se reúnam em pequenos refúgios que persistiram frente as ações humanas. Uma única árvore, por exemplo, pode abrigar no topo espécies de morcegos, em seu tronco, algum mamífero de médio porte e, próximo a base, roedores. Nesse micro-habitat, alguns patógenos podem, através de secreções fisiológicas, possuírem a oportunidade de "saltar" de uma espécie para outra, originando assim, um novo agente patológico (JOLY; QUEIROZ, 2020, TOLFO, 2020).

Para além disso, o tráfico de animais silvestres, assim como a produção intensiva de animais em locais inadequados, tem contribuído para alta taxa de destruição da diversidade e biológica e, dessa maneira, ao surgimento de diferentes patologias (FERREIRA, 2020).

A forma confinada e estressante com a qual os animais são manipulados nas duas situações citadas, colaboram para que não apenas os patógenos encontrem um ambiente adequado para realizar o transbordamento, como, também, de se disseminar nos animais que já estão debilitados, dada a situação de estresse sob a qual foram submetidas (FERREIRA, 2020, SOUZA, 2020).

Outra importante forma de viabilizar o surgimento de zoonoses, levantada pelos autores, é a aproximação indevida das pessoas e animais domésticos aos ambientes selvagens mediante a urbanização desordenada. Tal aproximação, viabiliza a troca de diferentes agentes patológicos possibilitando que esses patógenos passem a infectar seres humanos (SOARES et al., 2014).

Ademais, nesses locais a presença de vetores e reservatórios, tais como: caracóis, mosquitos, moscas, roedores e carrapatos, se dão com maior frequência, proporcionando não apenas ao aparecimento, mais ainda, o reaparecimento de importantes doenças, como: Febre amarela; Hantaviroses; Leishmanioses; leptospirose e schistosomose (BASTOS et al., 2016; MEDEIROS, 2019; SILVESTRINI PAES; CASTRO, 2020).

Dado ao apresentado é possível inferir que importantes estudos vêm sendo realizados no objetivo de compreender de que forma a dinâmica das doenças zoonóticas vem se dando. Contudo, observa-se, assim como no estudo realizado por Reis et al., (2016), que as doenças ditas negligenciadas continuam sendo negligenciadas, em especial para financiamento de estudos sobre elas.

A grande maioria das pesquisas realizadas se deram exatamente em momentos de crises sanitárias ou em quadros de potenciais surtos epidêmicos. Dessa forma, defende-se aqui que as patologias devam ser investigadas em sua completitude, independente da população que seja mais acometida ou qual classe social mais padeça.

Os patógenos desconhecem limites territoriais e linhas imaginárias criadas pelos homens para separar as nações, estados ou bairros. É necessário, portanto, ser compreendido que as enfermidades, em especial as negligenciadas, possuem considerável potencial de desenvolver epidemias, e, por consequente, demandam atenção tanto da academia quanto governamental.

\subsubsection{Aumento no número de doenças devido mudanças climáticas}

Trabalhos como os realizados por Sette e Ribeiro (2011); Artaxo (2020); Nascimento et al. (2021); Valdvino, Rodrigues e Coelho (2021), vem demonstrando que um dos principias impactos gerados na saúde humana pela degradação da biodiversidade, é o aumento de doenças e mortes provenientes das mudanças climáticas.

O desmatamento intenso, o consumo cada vez maior de recursos naturais, a utilização persistente de combustíveis fósseis e a liberação de gases que intensificam o efeito estufa, tem contribuído de maneira determinante para o aquecimento global ocorra de forma vertiginosamente (FERREIRA; PEIXOTO, 2020). Como consequência a esse aumento na velocidade do aquecimento, uma série de catástrofes vem se tornando corriqueiras (ARTAXO, 2020).

De acordo Silva, Xavier e Rocha (2021) o número de fenômenos naturais catastróficos como furacões, tornados, inundações, períodos de seca, onda de calor e frio extremo, queimadas e irregularidade pluviométrica tem ocorrido cada vez mais constantemente nas últimas décadas, levando milhares pessoas a morte todos os anos.

Além disso, graças a irregularidades no regime de chuvas e ao aquecimento gradativo dos ecossistemas, importantes dinâmicas ecológicas têm sido perturbadas, sobretudo, aqueles que envolvem artrópodes e pequenos roedores (ASSAD, 2016).

A flutuação irregular climática existente tem levado a erupção populacional de mosquitos, como o Aedes, vetor de inúmeras patologias virais, consequentemente é observado um aumento no número de caso de dengue, Zika, Chikungunya e febre amarela (CAMPOS et al., 2017). Outro vetor que tem sua dinâmica populacional alterada graças a alterações no clima é o Anopheles, fundamental na cadeia de transmissão 
da malária, essa doença é responsável por mais cerca de um milhão de mortes anuais (LIMA; MOREIRA; NÓBREGA, 2016); (CAMPOS et al., 2018).

Vale salientar que o aumento na incidência de doenças possuintes, em seus ciclos, de reservatórios naturais, é influenciado de maneira importante pelas mudanças climáticas. Dado o fato dessas alterações no clima levarem a flutuações no provimento de recursos para esses animais (reservatórios), que podem em um momento de grande abundância se proliferar e, por consequência induzirem surtos epidêmicos, ou, quando contrário, em quadros de escassez extrema esses organismos podem se aproximar dos ambientes urbanos/domésticos acarretando numa maior possibilidade de contagio entre o patógeno presente naturalmente nesses reservatórios naturais e seres humanos. Exemplo disso é foi o surto ocasionado pela doença de Lyme nos Estados Unidos e por hantaviroses na América do Sul (ESTELLITA-LINS, 2020); (LIMA et al., 2021).

Importante consequência das alterações climáticas é a expansão do nicho de vetores e reservatórios naturais de importantes doenças tais como arboviroses - viroses transmitidas por artrópodes (MATIAS; MASTEGHIN; IMPERADOR, 2020). Graças ao aumento na temperatura global e as irregularidades pluviométrica em todo o planeta, diversos animais que atuam como meio de transmissão ou reservatórios dos patógenos, por exemplo, mosquitos e carrapatos, como acima citado, encontram condições adequadas para sua sobrevivência em locais outrora inóspitos (MEASON; PATERSON, 2014).

Ademais as fortes ondas de calor e frio, consequência da desregulação meteorológica/climática, contribuem substancialmente para o agravamento das doenças do sistema nervoso, cardiopulmonares, e patologias relacionadas a reações de hipersensibilidade se (BORDIN; SILVA; PEREHOUSKEI, 2017, FURTADO et al., 2019). Tendo em vista que alterações no ambiente, reflete incisivamente na fisiologia não apenas humana, mas de todos os organismos (SETTE; RIBEIRO, 2011, JESUS, 2019).

Segundo o Painel Intergovernamental de Mudanças Climáticas (IPCC), em seu AR4, o Aumento da morbidade e mortalidade por ondas de calor, com ênfase no evento de 2003 no verão Europeu, vitimou fatalmente cerca de 32.000 pessoas, a maioria, idosos, no início dos anos 2000 (IPCC, 2008).

Frente a isso é possível perceber que as alterações no clima reverberam de maneira intensa na saúde da população humana. Quando investigado os principais motivos causadores das alterações bruscas que vem ocorrendo nas condições climáticas e meteorológicas, poder-se-á inferir a destruição da biodiversidade como sendo um dos principais fatores, como demonstrado nos dados acima (BERTOLAMI; FRANCISCO, 2020).

No entanto, vale observar que o número de estudos é, ainda, muito pequeno dada a complexidade que as abordagens referentes as mudanças climáticas e a sua correlação com saúde da população, sobretudo nas regiões tropicais. Dessa forma, muito dos danos causados por essas abruptas alterações climáticas, não estão sendo investigadas, sendo de urgente necessidade que a academia e autoridades competentes passem a olhar com mais atenção para esse problema.

\subsubsection{Emergência e/ou re-emergência de enfermidades provenientes da poluição}

O terceiro fator relacionado a perda da biodiversidade e impactos na saúde humana, apresentado pelos autores nos trabalhos analisados nessa pesquisa, foi a relação entre o aumento da poluição e a emergência e/ou re-emergência de doenças.

Segundo a Organização Mundial da Saúde, no ano de 2016, 12,6 milhões de pessoas perderam suas vidas por morarem ou trabalharem em ambientes poluídos. Para além das doenças infeciosas adquiridas por pessoas resistentes nesses locais, há, ainda, a exposição a tóxicos e radiação que colabora para o adoecimento de milhares de pessoas e outros tantos venham a óbito.

Contudo, nesse trabalho, irá ser destacada apenas as doenças infecciosas relacionadas a poluição, tendo em vista que essa é uma consequência direta da perturbação no equilíbrio ecológico dos ecossistemas que estão sendo poluídos (RIBEIRO, 2019).

Muito da poluição dos habitats, terrestres ou aquáticos, se deve, dentre outras coisas, ao despejo de dejetos ou substâncias artificias de maneira indiscriminada e sem os cumprimentos dos protocolos vigentes (TESTA; ROSA; FAVERO, 2020). Outro grande responsável por poluir os ecossistemas é a urbanização desorganizada e irregular (SELANI, 2020).

Com o aumento de dejetos no solo e, principalmente, nos rios e oceanos, toda a estrutura do sistema ecológico é alterada, uma das primeiras modificações é a exclusão de espécies que dependem de um ambiente com condições muito especificas e, por consequência, a dominância de espécies mais generalista (MELO; JOHNSSON, 2018); (KOERICH et al., 2021). Esses organismos que possuem esse perfil mais generalista, são, também, importantes componentes no ciclo de inúmeras patologias, por exemplo, leptospirose, leishmaniose e verminoses (SILVA; LINS; CASTRO 2016)

A biodiversidade existente em ambientes poluídos é escassa e deveras características. Nesses locais são encontrados roedores, moscas, mosquitos e moluscos como os caracóis (BRAZ; LONGO, 2019). Todos esses agrupamentos de animais possuem representantes que atuam diretamente na transmissão de doenças (BRAZ; LONGO, 2019).

Outro importante impacto da poluição na biodiversidade que desemboca em desordens patológicas nos seres humanos, é o aumento de matéria orgânica nos oceanos por meio do despejo de esgoto. Esse súbito acréscimo de compostos orgânicos leva a proliferação de determinadas algas, como os dinoflagelados, como consequência esses organismos passam 
a liberar toxinas, a fim de controlar o tamanho populacional, contudo outros animais podem ingerir essas substâncias, realizando o processo de biomagnificação (VALE, 2019). O resultado é que quando esses animais são consumidos pelos seres humanos, devido a grande quantidade de toxinas neles acumulados, são desenvolvidos quadros clínicos graves podendo evoluir ao óbito (VALE, 2019).

Portanto, infere-se que os danos causados pela poluição incidem de maneira direta sobre a saúde da população. Registra-se aqui, que, assim como nos trabalhos de Reis et al. (2016) e Monteiro e Silva (2017), notou-se que as populações mais impactadas pelas consequências proveniente tanto da poluição, quanto dos outros tópicos descritos, são aquelas que se encontram em situação de vulnerabilidade socioeconômica.

Dessa forma, é necessário que medidas sejam tomadas tanto no âmbito sanitário quanto social e econômico, a fim de que as pessoas situadas em zonas de risco sejam alocadas, ou minimamente assistidas, para ser assegurado o direito à vida, vida essa com dignidade.

Dado ao exposto, é notória a importância da biodiversidade e como a manutenção de sua integridade é fundamental para ser atingido o completo estado de bem-estar que configura o conceito de saúde. Em contrapartida, faz-se necessário estudos relacionados a doenças que emergiram ou reapareceram mediante declínio da biodiversidade ocorra com mais intensidade e de maneira contínua, especialmente nos ambientes tropicais onde a lacuna no conhecimento é maior.

Salienta-se, ainda, a necessidade de uma abordagem específica relacionada não apenas aos impactos, mais, também a proposição de medidas objetivando a diminuição dos impactos às populações em situação de risco.

\section{Conclusão}

O estado saudável da população humana depende fundamentalmente da integridade do ambiente e da biodiversidade. É possível perceber, de acordo ao apresentado nesse trabalho, que importantes reverberações e impactos são gerados a saúde do homem mediante o declínio da diversidade biológica.

Nessa pesquisa, constatou-se que os principais impactos relacionados a crise da biodiversidade à saúde humana de acordo diferente autores, são o aumento no número de doenças zoonóticas e o crescimento no número de pessoas que adoeceram direta, ou indiretamente, devido a mudanças climáticas e a emergência e/ou re-emergência de enfermidades provenientes da poluição.

Dessa forma, conclui-se, sugerindo que as políticas conservacionistas sejam incorporadas as medidas de saúde global, e a necessidade de mais trabalhos sejam desenvolvidos no intuído de não apenas compreender, mais, também, desenvolver medidas contra os motivos originários do surgimento e o ressurgimento das diferentes patologias e que os estudos, assim como medidas legais sejam tomadas a fim de minimizar os danos que as populações em situação de vulnerabilidade sanitária e socioeconômica vêm sofrendo devido a degradação da diversidade biológica.

\section{Referências}

ALHO, C.J. Importância da biodiversidade para a saúde humana: uma perspectiva ecológica. Estudos Avançados, v.26, p.151-166, 2012. doi: 10.1590/S0103-40142012000100011

AMARAL, C.P. Zoonoses x mutações: qual a relação com as questões ambientais? RevBEA, v 15, n 4, p.310-326, 2020. doi: 10.34024/revbea.2020.v15.10619

ARTAXO, P. As três emergências que nossa sociedade enfrenta: saúde, biodiversidade e mudanças climáticas. Estudos Avançados, v. 34, p. 53-66, 2020. doi: 10.1590/s0103-4014.2020.34100.005

ASSAD, L. Relações perigosas: aumento de temperatura e doenças negligenciadas. Ciênc. Cultura, v. 68, n.1, p.14-16, 2016. doi: 10.21800/2317-66602016000100007

BASTOS, T. S. A. et al. Carrapatos em animais silvestres do bioma cerrado triados pelo cetas, IBAMA-Goiás. Ciênc. Anim. Bras., v.17, p.296-302, 2016. doi: 10.1590/1089-6891v17i236239

BERTOLAMI, O.; FRANCISCO, F. Alterações climáticas e o Sistema Terrestre. Rev. Ciênc. Elementar, v.8, n.4, p.1- 6, 2020. doi: $10.24927 /$ rce2020.051

BORDIN, R.O. et al. Análise espacial com foco na poluição atmosférica e suas consequências na saúde respiratória em Rondonópolis, mato grosso. Biodiversidade, v.16, n.2, p.98-111, 2017.

BRAZ, S.N.; LONGO, R.M. Bioindicadores de Poluição Ambiental: Um Estudo Bibliométrico. Rev. Cient. ANAP Brasil, v.12, n.27, p.42-53, 2019. doi: 10.17271/19843240122720192239

CAMPONOGARA, S. Saúde e meio ambiente na contemporaneidade: o necessário resgate do legado de Florence Nightingale. Esc. Anna Nery, v.16, p.178-184, 2012. doi: 10.1590/S1414-81452012000100024

CAMPOS, F. I. et al Meio ambiente, Desenvolvimento e expansão de doenças transmitidas por vetores. Fronteiras J. Soc. Technol. Environ. Scie., v.7, n.2, p.49-63, 2018.

CNDSS - Comissão Nacional Sobre os Determinantes Sociais da Saúde. Carta aberta aos candidatos à Presidência da República. 2006. Disponível em: www.determinantes.fiocruz.br. Acesso em: 11 maio 2021

ESTELLITA-LINS, C. Exfermidade, enfermidade ou experiência de doença: zoonose e antropoceno. O que nos faz. Pensar, v.29, n.47, p.192-237, 2020. doi: 10.32334/oqnfp.2020n47a741.

FERREIRA, J.M.; BARROS, N.M. O tráfico de fauna silvestre no Brasil e seus impactos. Direito Penal Processo Penal, v. 2, n. 2, p. 76-100, 2020.

FERREIRA, M.L.; PEIXOTO, B.T. Coronavírus e direito ambiental: necessária discussão para a superação de uma crise humana e ecológica. Rev. Jurídica FA7, v.17, n.3, p.87-108, 2020.

FILHO, M.F. Devem os animais serem culpados pelas pandemias? Uma análise de contexto. Rev. Espaço Crítico, v.1, n.1, p.41-60, 2020.

FURTADO, B.L. et al. Efeitos à saúde humana da intensificação da poluição atmosférica causada por queimadas. Biodivers. Bras.-BioBrasil, n.1, p.125-125, 2019.

GOMES, A.S; NETO, J.D.; SILVA, V.F. Serviços ecossistêmicos: conceitos e classificação. Rev. Iberoam. Ciênc. Amb., v.9, n.4, p.12-23, 2018. doi: 10.6008/CBPC2179-6858.2018.004.0002 
IPCC - Intergovernmental Panel on Climate Change. Climate change 2008: IPCC four Assessment; a report of the Intergovernmental Panel on Climate Change. 2008. Disponível em: < https://www.ipcc.ch/site/assets/uploads/2018/02/ar4_syr_ full_report.pdf $>$. Acesso em: 28 jun. 2021.

JESUS, S.J.A. A saúde humana e o meio ambiente frente aos processos de degradação: uma revisão da literatura. Rev. Interfaces Saúde Hum. Tecnol., v.7, n 2, p.297-304, 2019. doi: 10.16891/2317-434X.v7.e2.a2019.pp297-304

JOLY, C.A.; QUEIROZ, H.L. Pandemia, biodiversidade, mudanças globais e bem-estar humano. Estud. Avan., v.34, p.6782, 2020. doi: 10.1590/s0103-4014.2020.34100.006

KOERICH, P. et al. Determinação de contaminantes emergentes no rio lontra (salto do lontra-paraná). Biodiversidade, v.20, n.1, p.1-16, 2021.

LIMA, R.C; MOREIRA, E.B.M.; NÓBREGA, R.S. A influência climática sobre a epidemia dengue na cidade do Recife por Sistema de Informações Geográficas. Rev. Bras. Geografia Fís., v.9, n.2, p.384-398, 2016. doi: 10.26848/rbgf.v9.2.p384-398

LIMA, T.F.S. et al. Variantes climáticas e sua relação com as doenças de origem infecciosa: uma revisão integrativa. Res. Soc. Develop., v.10, n.2, p.1-11, 2021. doi: 10.33448/rsd-v10i2.12126

MATIAS, T. P.; MASTEGHIN, L. T.; IMPERADOR, A. M. A Sustentabilidade ambiental: da utopia à emergência. $\operatorname{Rev} B E A$, v.15, n.4, p.160-174, 2020. doi: 10.34024/revbea.2020.v15.10830

MEASON, B.; PATERSON, R. Chikungunya, climate change, and human rights. Health Hum. Rights, v.16, n.1, p.105-112, 2014.

MEDEIROS, J. Manejo integrado de vetores: uso de telas milimétricas em janelas. J. Biol. Pharm. Agricul. Manag., v.15, n.2, p.156-168, 2019.

MELO, M.C.; JOHNSSON, R.M.F. O conceito emergente de segurança hídrica. Sustentare, v.1, n.1, p.72-92, 2018. doi: 10.5892/st.v1i1.4325

METZGER, J.P.; CASATTI, L. Do diagnóstico à conservação da biodiversidade: o estado da arte do programa BIOTA/ FAPESP. Biota Neotrop., v.6, p.1-14, 2006. doi: 10.1590/S167606032006000200002

MONTEIRO, A.F.; SILVA, C.L. Saúde pública no brasil: mapeamento histórico através de recorte literário. Biodiversidade, v.16, n.2, 2017.

MONTEIRO, M.R. Promoção da saúde: recurso hídrico, educação, saúde e meio ambiente para a prática da cidadania no interior do Amazonas. Rev. Sustinere, v.5, n.1, p.5-23, 2017. doi: 10.12957/sustinere.2017.27825

NASCIMENTO, R.Z. et al. Meio ambiente e a sua propagação da COVID-19. Braz. J. Develop., v.7, n.1, p.6888-6900, 2021.

OLLAIK, L.G.; ZILLER, H.M. Concepções de validade em pesquisas qualitativas. Educ. Pesq., v.38, p.229-242, 2012. doi: 10.1590/S1517-97022012005000002

OMS - Organização Mundial da Saúde. 10 fatos sobre a prevenção de doenças em ambientes saudáveis, março de 2016. Disponível em: $<$ https://www.who.int/features/factfiles/environmentaldisease-burden/en/> Acesso em: 26 jun. 2021.

PATIAS, N.D.; HOHENDORFF, J.V. Critérios de qualidade para artigos de pesquisa qualitativa. Psicol. Estudo, v.24, p.1-14, 2019. doi: $10.4025 /$ psicolestud.v24i0.43536

PEREIRA, A.C.A.; NARCISO, B.B.D.; MANSANO, C.F.M. Doenças Infecciosas Emergentes e Re-emergentes: Emerging and Re-emerging Infectious Diseases. Arch. Health, v.2, n.4, p.725-
$728,2021$.

RABELO, A.M.; OLIVEIRA, D.B. Impactos ambientais antrópicos e o surgimento de pandemias. Unifesspa, v.26, p.1-7, 2020.

REIS, A.C.S.M. et al. O cenário de políticas públicas do Brasil diante do quadro das doenças negligenciadas. Saúde Ciênc. Ação, v.2, n.2, p.99-107, 2016.

RIBEIRO, H. Poluição, um veneno silencioso para a saúde humana. Rev. Ciênc. Elementar, v.7, n. 4, p.1-5, 2019. doi: $10.24927 /$ rce2019.069

RIBEIRO, A.C.A. et al. Zoonoses e Educação em Saúde: Conhecer, Compartilhar e Multiplicar. Braz. J. Health Rev., v.3, n.5, p.12785-12801, 2020.

SANTOS, C.A. et al. Queimadas e seus impactos no ecossistema e na saúde da população. Rev. Trab. Acad. Universo Recife, v.5, n.2, p.1-3, 2019.

SELANI, R.L. Cenários patológicos da urbanização em Santa Cruz do Rio Pardo, São Paulo. Seminário Nacional-Urbanismo, Tempo e Espaço, v.1, n.1, p.1-5, 2020.

SETTE, D.M.; RIBEIRO, H. Interações entre o clima, o tempo e a saúde humana. InterfacEHS-Rev. Saúde, Meio Amb. Sustentab., v.6, n.2, p.37-51, 2011.

SILVA, A.A.L. Impactos de mudanças do uso da terra sobre a saúde humana. Ciênc. Cultura, v.73, p.25-29, 2021. doi: 10.21800/2317-66602021000100005.

SILVA, E.S.; LINS, G.A.; CASTRO, E.M.N.V. Historicidade e olhares sobre o processo saúde-doença: uma nova percepção. Rev. Sustinere, v.4, n.2, p.171-186, 2016. doi: 10.21800/231766602021000100005

SILVA, M.A.; XAVIER, D.R.; ROCHA, V. Do global ao local: desafios para redução de riscos à saúde relacionados com mudanças climáticas, desastre e Emergências em Saúde Pública. Saúde Debate, v.44, p.48-68, 2021. doi: 10.1590/010311042020 E204

SILVESTRINI, A.R.; PAES, A.C.; CASTRO, A.M.M.G. Emergência de zoonoses em desastres: relato de caso de leptospirose em Brumadinho, Minas Gerais. PUBVET, v.15, p.18, 2020. doi: 10.31533/pubvet.v14n01a735.

SIMÕES, M. et al. Integridade ecossistêmica da Amazônia brasileira através do uso de redes bayesianas e suas relações com os serviços ecossistêmicos In: SIMPÓSIO BRASILEIRO DE SENSORIAMENTO REMOTO, 17., 2015, João Pessoa. Anais... São José dos Campos: INPE, 2015. p. 5240-5247, 2015.

SINISGALLI, P.A.A; SOUSA JUNIOR, W.C. Serviços ecossistêmicos. Diál. Socioamb. Macrometrópole Paul., v.3, n.7, p.5-7, 2020.

SOARES, J.A.S. et al. Impactos da urbanização desordenada na saúde pública: leptospirose e infraestrutura urbana. Polêm! ca, v.13, n.1, p.1006-1020, 2014. doi: 10.12957/polemica.2014.9632

SOARES, T.O. et al. Impactos ambientais causados pelo desmatamento: uma revisão sistemática da literatura. Rev. Saúde Meio Amb., v.9, n.2, p.66-73, 2019.

SOUZA, L.L. Comer Animais e Zoonoses: utilidade da pecuária industrial. Voluntas Rev. Int. Filos., v.11, n.1, p.1-10, 2020. doi: $10.5902 / 2179378643987$

SOUZA, I.S.M. Fatores relacionados às zoonoses emergentes: revisão bibliográfica. Rev. Multidisc. Saúde, v.2, n. 1, p.87-87, 2021. doi: $10.51161 / \mathrm{rems} / 903$

TESTA, P.A.; ROSA, K.R.; FAVERO, L. Biodiversidade: 
principais ameaças e alertas. RETEC, v.13, n.1, p.29-35, 2020.

TOLFO, R. A destruição da natureza e a emergência de zoonoses: os temas híbridos e o contrato natural podem nos mostrar caminhos para superar problemas?. Em Construção, n.8, p.203209, 2020. doi: 10.12957/emconstrucao.2020.54059

TRIVELLATO, G.M.L.; SANTOS, M.A. O papel dos seres humanos na manutenção dos ecossistemas. Rev. Relicário, v.6,n.11, p.104-116, 2019. doi: 10.46731/RELICARIO-v6n11-2019-121 VALDVINO, M.; RODRIGUES, F.; COELHO, P. Alterações climáticas e zoonoses: influência das alterações climáticas na propagação de doenças infeciosas. Higeia, v.5, n.1, p.41-52, 2021.

VALE, P. Intoxicação paralisante por marisco: uma síndrome rara com recorrência decadal?. Med. Interna, v.26, n.4, p.326-334, 2019. doi: 10.24950/rspmi/Revisao/96/19/4/2019

ZANELLA, J.R.C. Zoonoses emergentes e reemergentes e sua importância para saúde e produção animal. Pesq. Agropec. Bras., v.51,p.510-519, 2016. doi: 10.1590/S0100-204X2016000500011. 